\title{
Cross-sectional and prospective relationships of passive and mentally active sedentary behaviours and physical activity with depression - CORRIGENDUM
}

Mats Hallgren, Thi-Thuy-Dung Nguyen, Neville Owen, Brendon Stubbs, Davy Vancampfort, Andreas Lundin, David Dunstan, Rino Bellocco and Ylva Trolle Lagerros

https://doi.org/10.1192/bjp.2019.60, Published by Cambridge University Press, 21 March 2019

Keywords: Sedentary behaviour, physical activity, depression, isotemporal substitution modelling, corrigendum

The article currently states that 'walking' was included in the calculation of moderate-to-vigorous physical activity (MVPA). This is incorrect. Walking was categorised separately as 'light physical activity', while 'Strenuous exercise (e.g. jogging, swimming)' and 'hard training/competition' were combined to form the MVPA category, as otherwise described.

The authors wish to apologise for any inconvenience caused.
Mats Hallgren; Thi-Thuy-Dung Nguyen; Neville Owen; Brendon Stubbs; Davy Vancampfort; Andreas Lundin; David Dunstan; Rino Bellocco; YlvaTrolle Lagerros

doi:10.1192/bjp.2019.87

\section{Reference}

Hallgren M, Nguyen T-T-D, Owen N, Stubbs B, Vancampfort D, Lundin A, Dunstan D, Bellocco R and Lagerros YT. Cross-sectional and prospective relationships of passive and mentally active sedentary behaviours and physical activity with depression. $\mathrm{Br} J$ Psychiatry 2019; 1-7.

\section{Retraction}

\section{Association between particulate matter air pollution and risk of depression and suicide: systematic review and meta-analysis - RETRACTION}

Xuelin Gu, Qisijing Liu, Furong Deng, Xueqin Wang, Hualiang Lin, Xinbiao Guo and Shaowei Wu

DOI: https://doi.org/10.1192/bjp.2018.295, Published online by Cambridge University Press, 05 February 2019

Keywords: Particulate matter; depression; suicide; meta-analysis; retraction

We, the Editors of the British Journal of Psychiatry, have retracted the following article: $\mathrm{Gu}$ et al, 2019.

In January 2020, the authors alerted the editorial team to an error in the analyses. The authors transformed the effect estimates extracted from all included studies to the same scale (per $10 \mu \mathrm{g} / \mathrm{m}^{3}$ increase in particulate matter). The error occurred during the data transformation for the $95 \%$ confidence intervals of the extracted effect estimates. The authors inappropriately expanded the standard errors of some effect estimates during the log and antilog changes.

Following a detailed investigation by the senior editorial committee, it was identified that the main findings are unreliable as a result of the error. The corrected analyses no longer indicate an association between $\mathrm{PM}_{2.5}$ and depression and the association with depression at either exposure $\left(\mathrm{PM}_{2.5}\right.$ and $\left.\mathrm{PM}_{10}\right)$ are close to identical.

The authors agree to the retraction and the Editors would like to thank the authors for alerting the editorial team to the error.

Xuelin Gu; Qisijing Liu; Furong Deng; Xueqin Wang; Hualiang Lin; Xinbiao Guo Shaowei Wu

doi:10.1192/bjp.2020.87

\section{Reference}

1. Gu X, Liu Q, Deng F, Wang X, Lin H, Guo X and Wu S. Association between particulate matter air pollution and risk of depression and suicide: systematic review and meta-analysis. Br J Psychiatry 2019; 215: 456-467. doi: 10.1192/ bjp.2018.295. 